\title{
HACIA LA SEGURIDAD ALIMENTARIA EN CONTEXTOS DE CAMBIO CLIMÁTICO EN CLAVE DE GOBERNANZA GLOBAL*
}

\section{Towards Food Security in contexts of Climate Change in a Global Governance key}

\author{
ANDREa LUCAS GaríN** \\ María Constanza Cubillos Torres*** \\ Universidad Finis Terrae \\ Santiago, Chile
}

\footnotetext{
* Los resultados preliminares fueron expuestos en las XLVI Jornadas Chilenas de Derecho Público "Hacia una nueva Forma de Gobierno y Administración. Perspectivas nacionales e internacionales", Ilevadas a cabo los días 17 y 18 de noviembre de 2016 en la Facultad de Derecho de la Universidad de Chile, en la Comisión Derecho Internacional. Este trabajo se enmarca en el desarrollo del Proyecto FONDECYT de Iniciación 2015 Nro. 11150382 titulado "Instrumentos comerciales para enfrentar el cambio climático en Chile: vínculos con el sistema internacional de comercio".

** Doctora en Derecho y Ciencias Sociales y LLM in International Law. Profesora Investigadora Universidad Finis Terrae, Av. Pedro de Valdivia 1509, Facultad de Derecho de la Universidad Finis Terrae. Santiago de Chile, Chile. Correo electrónico: <andrealucasg@ hotmail.com>.

*** Médico Veterinario y Asistente de Investigación de la Universidad Finis Terrae. Av. Pedro de Valdivia 1509, Facultad de Derecho de la Universidad Finis Terrae. Santiago de Chile, Chile. Correo electrónico: <mcubillost@uft.edu>.
}

Artículo recibido el 12 de diciembre de 2016 y aceptado para publicación el 30 de marzo de 2017. 
RESUMEN: En el contexto del Sistema de Protección Climática y de su más reciente tratado multilateral, el Acuerdo de París, el trabajo inicia con un bosquejo de la Gobernanza Global como un escenario donde se centra la investigación, para ocuparnos a continuación de la Seguridad Alimentaria a fin de conceptualizarla, luego abordaremos el Sistema Climático y los impactos específicos que tendrá sobre la Seguridad Alimentaria en esta región latinoamericana; se revisarán las inclusiones sobre Seguridad Alimentaria que el Acuerdo de París previó; se examinarán los pasos que está dando Chile en la materia, para finalizar con algunas medidas específicas que los Estados debieran ir emprendiendo, de modo de ir asegurando la Seguridad Alimentaria, como parte de sus compromisos asumidos en el Acuerdo de París. Se aportarán conclusiones en clave de Gobernanza Global.

PALABRAS CLAVE: Seguridad Alimentaria, Cambio Climático, Acuerdo de París, Gobernanza Global

\begin{abstract}
In the context of the Climate Protection System and its most recent multilateral treaty, the Paris Agreement, this paper begins with an outline of Global Governance as a scenario where research is focused, in order to conceptualize what is Food Security. Then we will address the Climate System and the specific impacts it will have on Food Security in the Latin American region; the Food Security inclusions envisaged by the Paris Agreement will be reviewed; the steps being taken by Chile in this area will be examined in order to finalize proposing some specific measures that States should undertake in order to ensure Food Security as part of their commitments under the Paris Agreement. Key findings from Global Governance will be provided.
\end{abstract}

KEYWORDS: Food Security, Climate Change, Paris Agreement, Global Governance

\title{
INTRODUCCIÓN: ESBOZANDO LOS CARACTERES DE LA GOBERNANZA GLOBAL
}

Siguiendo la tendencia desarrollada en la "era de los objetivos"1, en 1992 la Convención Marco de Naciones Unidas sobre el Cambio Climático se planteó a la Seguridad Alimentaria como una de las finalidades propias

En palabras de la Secretaria Ejecutiva de la CEPAL. Ocampo (2015) p. 11. 
del Sistema de Protección de Cambio Climático ${ }^{2}$, hoy con más de 22 años de vigencia.

El Cambio Climático continúa siendo una materia que ha atrapado la atención internacional, conformando un sistema que se ve enriquecido por las informaciones científicas que el Grupo Intergubernamental de Cambio Climático (PICC en adelante) renueva, y por las continuas negociaciones climáticas multilaterales. El Sistema de Protección Internacional se encuentra revitalizado a la luz del Acuerdo de París, vigente desde el 4 de noviembre de 2016, el primer gran acuerdo ambiental del Siglo XXI.

Dentro de las temáticas relacionadas, la Seguridad Alimentaria se presenta como un concepto nacido a instancias de la Organización de las Naciones Unidas para la Alimentación y la Agricultura (en adelante FAO), que ha procurado que los Estados la atiendan, atenta a que la lucha contra el hambre y la pobreza resultan un objetivo relevante.

Pensamos que tanto el Cambio Climático como la Seguridad Alimentaria se interceptan y son ejemplos de Gobernanza Global, es decir, de cómo los procesos y las instituciones internacionales conllevan impactos sobre los sistemas jurídicos; y son bienes públicos que influyen en las actividades que deben Ilevar adelante los Estados, pero que también tienen efectos sobre organizaciones internacionales, organizaciones no gubernamentales (ONGs), individuos, etc.

En el contexto del fenómeno climático y de su más reciente tratado multilateral, el Acuerdo de París, iniciaremos con un bosquejo de la Gobernanza Global como un escenario donde quisiéramos centrar nuestro trabajo, para ocuparnos a continuación de la Seguridad Alimentaria a fin de conceptualizarla y distinguirla de otros términos relacionados con el Derecho a la Alimentación, luego abordaremos el Sistema Climático y los impactos específicos que tendrá sobre la Seguridad Alimentaria en esta región latinoamericana; se revisarán las inclusiones sobre Seguridad Alimentaria que el

Desde su Artículo 2 Objetivo: El objetivo último de la presente Convención y de todo instrumento jurídico conexo que adopte la Conferencia de las Partes, es lograr, de conformidad con las disposiciones pertinentes de la Convención, la estabilización de las concentraciones de gases de efecto invernadero en la atmósfera a un nivel que impida interferencias antropógenas peligrosas en el sistema climático. Ese nivel debería lograrse en un plazo suficiente para permitir que los ecosistemas se adapten naturalmente al cambio climático, asegurar que la producción de alimentos no se vea amenazada y permitir que el desarrollo económico prosiga de manera sostenible (CMNUCC, el énfasis es nuestro). 
Acuerdo de París previó; se examinarán los pasos que ha dado Chile en la materia, para finalizar con algunas medidas específicas que los Estados debieran considerar, de modo de ir asegurando la Seguridad Alimentaria, como parte de sus compromisos asumidos en el Acuerdo de París. Las conclusiones recogerán algunas ideas en clave de Gobernanza Global.

Para el Derecho Ambiental, la cooperación internacional es una herramienta prioritaria que siempre ha utilizado desde sus inicios, allá por la década del setenta. En términos más actuales, la cooperación internacional se ve renovada por las perspectivas que la relacionan con la Gobernanza Global. En este apartado esbozaremos ${ }^{3}$ algunas características de la Gobernanza Global y las proyectaremos sobre las temáticas Seguridad Alimentaria y Cambio Climático.

En cuanto a los fundamentos de la Gobernanza Global, "[...] dicho concepto alude a una serie de transformaciones vinculadas a la globalización, que han afectado el entorno y las capacidades de los Estados nacionales". ${ }^{4}$ Esto se conecta con la globalidad del Cambio Climático, en cuanto a un fenómeno del que no escapa ningún Estado ni población (como ratificaremos al ocuparnos de sus impactos), como un problema global que activa la demanda de mayor internacionalidad y de más específicas regulaciones, según Anne Peters. ${ }^{5}$

Como primer adelanto de sus elementos, coincidimos con Serna de la Garza, que explica que la Gobernanza Global envuelve complejas interacciones entre Estados, organizaciones intergubernamentales y actores no-estatales de diversa índole (como empresas transnacionales y ONGs internacionales). ${ }^{6}$

Veamos a continuación los cuatro rasgos que caracterizan a la Gobernanza Global, conforme nos ilustran Von Bogdandy, Dann y Goldmann7. El primer rasgo presenta a la Gobernanza como un tema no solo de actores públicos (reconoce la importancia de las organizaciones internacionales y también destaca la relevancia de los instrumentos de carácter privado o híbrido, así como los emitidos por particulares); el segundo muestra que la informalidad es más aceptada (atento a que muchas instituciones, procedimientos e instrumentos

3 Esbozaremos estos temas dado que tienen muchas derivaciones en el Derecho Internacional, como las de Autoridad Pública, Fragmentación, etc.

4 Serna De la Garza (2009) p. 24.

5 Peters (2016) p. 3.

6 Serna de la Garza (2009) p. 26.

7 Cfr. Von Bogdandy et al. (2008) p. 1378. 
escapan a la comprensión de los conceptos legales establecidos); el tercero indica que pensar en términos de Gobernanza Global significa cambiar el enfoque de los actores a las estructuras y procedimientos; y el último se desprende del mismo uso del término "Global" en vez de "internacional": la Gobernanza Global hace hincapié en el enfoque multinivel (con lo que se supera la división entre fenómenos internacionales, supranacionales y nacionales).

A la luz de esos rasgos, podemos afirmar que la Seguridad Alimentaria es un concepto nacido al amparo de la FAO; por su parte, el Cambio Climático como fenómeno jurídico se ha desarrollado en el marco del Sistema de Protección de Cambio Climático bajo los auspicios del PNUMA ${ }^{8}$ centralmente y de la institucionalidad propia del Sistema, donde destacan las Conferencias de las Partes (COP), tanto de la Convención Marco de Naciones Unidas sobre el Cambio Climático como del Protocolo de Kioto. Asimismo, vemos un avance de "otros actores, como las empresas que están en un proceso de auto-responsabilización en el campo ecológico". ${ }^{9}$

Sobre el segundo atributo, es posible que algunas características de informalidad se vislumbren, justamente, en el modo en que los Estados Partes del Sistema de Cambio Climático se van obligando. Aun cuando no todos los instrumentos tienen la misma obligatoriedad, estos encuentros anuales han ido de a poco reforzando al Sistema y asegurando su vitalidad y actualidad.

En relación con la tercera característica, las estructuras y procedimientos son elementos claves para comprender el Sistema de Protección Climático, que ha nacido de un convenio marco, con dos protocolos que han continuado desarroIlándose en base a estas reuniones anuales que mencionamos, que también se han retroalimentado por las presencia de la sociedad civil, donde las ONG's han cumplido un rol informativo, consultivo, pero también multiplicador en la difusión del fenómeno y su concientización, junto con el de reforzar la presencia de estas temáticas en las agendas públicas e incluso privadas. ${ }^{10}$

8 Pnuma son las siglas del Programa de las Naciones Unidas para el Medio Ambiente.

9 Según FrançOIS Ost: "La estrategia de la autor-responsabilización de las empresas en el campo ecológico consiste en 'interiorizar las externalidades producidas', como dicen los economistas, a la vez que se preserva su autonomía (REHBINDER, 1991, p. 598). Preocupadas por mejorar su imagen ante el gran público, teniendo en cuenta los efectos negativos de su acción sobre el medio natural a la vez que siguen controlando desde el interior el proceso normativo de reglamentación de esta acción, las empresas adoptan hoy día el discurso de la autorresponsabilidad", Ost (2006) p. 102.

10 Piénsese en la Responsabilidad Social Empresarial por ejemplo, y en el desarrollo de Códigos de Conductas en las empresas, como señalamos en la nota anterior. 
A la vista del cuarto rasgo, concordamos que la Gobernanza Global encierra un enfoque multinivel para el tratamiento de los tópicos, en el cual todos los niveles de gobierno (internacional, supranacional, regional, nacional y local) deben aunar sus esfuerzos para asumir los desafíos que la Seguridad Alimentaria y el Cambio Climático involucran.

En clave de regulaciones, hay un impulso a favor del desarrollo de estándares internacionales, que luego requieren ser traspasados al ámbito nacional por los Estados. ${ }^{11}$ Esto puede ser considerado una muestra del modo en que la Gobernanza Global es una realidad que avanza en el Derecho Internacional.

Pensamos que este requerimiento de internacionalidad que destacamos, no significa que el Estado es más débil, sino que el Estado no puede en solitario cumplir con las exigencias de las propias demandas de legitimidad a las que debe responder frente a sus sociedades nacionales. Ahí es donde el Derecho Internacional acude en su ayuda para apuntalarlo y acompañarlo.

Se ha señalado la necesidad de más Derecho Internacional para hacer frente a una serie de cuestiones que no son claramente internacionales, pero que adecuadamente pueden ser descriptas como globales. Los asuntos globales son problemas que surgen a nivel de todo el mundo, y para los cuales el Estado, como una unidad normativa en última instancia, se considera insuficiente. Ellos normalmente se ocupan de la regulación de los "bienes públicos" o "comunes globales". Algunas cuestiones "globales" son clásicos temas de Derecho Internacional que han de formularse en condiciones de globalización, por ejemplo, el Derecho del Mar se transforma gradualmente de una cuestión de competencia y libertades, a un régimen de intercambio de recursos, gestión de los recursos y la protección del medio ambiente. El Cambio Climático cae en esta categoría de "global". ${ }^{12}$

Desde la perspectiva del Derecho Internacional del Siglo XXI, frente a su gran desarrollo normativo y su ensanchamiento por las materias de las que se

11 Así lo ilustra Serna De La Garza (2009) pp. 29 y 30: "En primer lugar, los instrumentos de la OMC inciden sobre un gran número de materias reguladas por los órganos legislativos nacionales, que de alguna manera están vinculadas al comercio. Estas materias van desde los impuestos, incentivos fiscales, aranceles y subsidios, hasta normas oficiales, medio ambiente, medidas sanitarias, seguridad y propiedad intelectual, entre muchas otras. Esto significa que hay numerosos puntos de contacto entre las reglas de la OMC y el derecho nacional, en los cuales coinciden ambos, y en los que el derecho interno ha de ajustarse a los estándares del referido organismo intergubernamental".

12 Cfr. Cottier et al. (2014) p. 318. 
ocupa, se observa un re-enfocamiento que ha pasado de ocuparse de lo que ocurría fronteras afuera de los Estados, a ocuparse cada vez más de lo que ocurre en sus fronteras adentro. ${ }^{13}$ De un Derecho Internacional regulador de las relaciones entre los Estados, a un Derecho cuyo foco son las actividades dentro de los Estados. Un ejemplo lo veremos en este artículo, dado que la Seguridad Alimentaria y el Cambio Climático son temas nacidos al amparo del Derecho Internacional y que muestran una tendencia cada vez más intensa de regular lo que ocurre dentro de las fronteras estatales.

Por último, debe advertirse que el concepto de Gobernanza Global también ha sido puesto en dudas, principalmente por la legitimidad de las instituciones que generan esta normatividad y por la inseguridad que afecta la predictibilidad y unidad del Derecho Internacional. Al igual que Von Bogdandy, Dann y Goldmann ${ }^{14}$, vemos que la Gobernanza Global abre un camino para satisfacer la legitimidad contemporánea, donde ya no está solo el Estado, sino que también está acompañado por la comunidad internacional, representada por los tradicionales más los nuevos sujetos de la escena internacional.

\section{EN CONTEXTOS DE SEGURIDAD ALIMENTARIA}

\section{Distinciones en clave alimentaria}

Antes de esbozar un concepto de Seguridad Alimentaria, quisiéramos hacer mención de que el Derecho Internacional se ha ocupado del Derecho a la Alimentación, consagrándolo como un derecho humano ya desde la Declaración Universal de Derechos Humanos ${ }^{15}$, y con mayor precisión en

13 Con ideas similares MANGAS (2014) p. 32: “De esta forma, igualdad de derechos y la mejora de las condiciones de vida se ponen al servicio del valor por excelencia, entonces y hoy, la paz. Ahora bien, la ausencia de guerra no es suficiente, no es una situación sin más, sino que precisa de condiciones previas que la sustenten, la fortalezcan y la hagan duradera".

14 Cfr. Von Bogdandy et al. (2008) p. 1376.

15 Artículo 25: Toda persona tiene derecho a un nivel de vida adecuado que le asegure, así como a su familia, la salud y el bienestar, y en especial la alimentación, el vestido, la vivienda, la asistencia médica y los servicios sociales necesarios; tiene asimismo derecho a los seguros en caso de desempleo, enfermedad, invalidez, viudez, vejez u otros casos de pérdida de sus medios de subsistencia por circunstancias independientes de su voluntad. Hacemos presente que no seguiremos un abordaje desde los Derechos Humanos de la Seguridad Alimentaria. 
el Pacto Internacional de Derechos Económicos, Sociales y Culturales ${ }^{16}$, el cual explica con mayor detalles los compromisos que los Estados toman en relación con este derecho. ${ }^{17}$

La relación del Derecho a la Alimentación con la Seguridad Alimentaria consiste en que ésta es "[...] una condición necesaria para el disfrute del Derecho a la Alimentación, definiéndose como un Estado donde está garantizado el acceso a todas las personas, en todo momento a los alimentos necesarios, seguros y nutritivos para llevar una vida sana".$^{18}$

Elemento fundamental del Derecho a la Alimentación es el acceso a los alimentos. Éste último debe ser considerado como acceso físico y también económico, es decir disponiendo de los medios o recursos para obtener una alimentación apropiada. Ahora bien, y siguiendo la Observación General

16 Artículo 11: 1. Los Estados Partes en el presente Pacto reconocen el derecho de toda persona a un nivel de vida adecuado para sí y su familia, incluso alimentación, vestido y vivienda adecuados, y a una mejora continua de las condiciones de existencia. Los Estados Partes tomarán medidas apropiadas para asegurar la efectividad de este derecho, reconociendo a este efecto la importancia esencial de la cooperación internacional fundada en el libre consentimiento. 2. Los Estados Partes en el presente Pacto, reconociendo el derecho fundamental de toda persona a estar protegida contra el hambre, adoptarán, individualmente y mediante la cooperación internacional, las medidas, incluidos programas concretos, que se necesiten para: a) Mejorar los métodos de producción, conservación y distribución de alimentos mediante la plena utilización de los conocimientos técnicos y científicos, la divulgación de principios sobre nutrición y el perfeccionamiento o la reforma de los regímenes agrarios de modo que se logre la explotación y la utilización más eficaces de las riquezas naturales; b) Asegurar una distribución equitativa de los alimentos mundiales en relación con las necesidades, teniendo en cuenta los problemas que se plantean tanto a los países que importan productos alimenticios como a los que los exportan.

17 El Pacto de Derechos Económicos, Sociales y Culturales se viola cuando un Estado no garantiza la satisfacción de un nivel mínimo para que las personas no padezcan hambre. Así, el Comité del Pacto, debió definir, cuáles eran las obligaciones del Estado con respecto a este derecho: Ellas son respetar, proteger y satisfacer sus necesidades básicas alimentarias. Cfr. Gordillo y Méndez (2013) p.5. Y según lo indicado por el relator del Comité, si un individuo no fuese capaz de proveer la alimentación básica, los Estados deben otorgarla directamente. Cfr. "Relator Especial sobre el derecho a la alimentación", Oficina del Alto Comisionado para los Derechos Humanos (ACNUDH) (1996-2017). Disponible en: < http://www.ohchr.org/SP/Issues/Food/Pages/Foodlndex.aspx>, fecha de consulta: 4 de abril 2017.

18 Rodríguez-Chaves y Pérez (2015) p. 50. Esta perspectiva de Derechos Humanos no será parte de nuestro análisis. 
$\mathrm{n}^{\circ} 12$ del Comité de Derechos Económicos, Sociales y Culturales de Naciones Unidas $^{19}$, este derecho debe contemplar tanto la disponibilidad de alimentos como su calidad.

En consonancia con lo anterior, el Comité ha señalado que los Estados deben alcanzar una alimentación adecuada para todas las personas de manera progresiva, y por último, que no solo es el Estado el responsable de asegurar este derecho, sino la sociedad en su conjunto.

Un concepto parte del Derecho a la Alimentación, en el cual no nos centraremos, empero es necesario distinguir, es el de Soberanía Alimentaria, que básicamente es el derecho de los pueblos a que de manera autónoma elijan su política alimentaria y forma de utilizar sus recursos.

La Soberanía Alimentaria tiene su origen en movimientos campesinos que, en contra de la lógica de mercado, proponen terminar con el hambre desde una concepción agroecológica en torno a algunos pilares como son: alimentación para el pueblo, sistemas compatibles con la naturaleza, favorecimiento de la agricultura familiar campesina, control a favor del mercado local, entre otros.

El concepto tomó fuerza luego de ser planteado en la Cumbre de Alimentación en Roma (1996). Sus alcances son amplios, y lo interesante que tiene es el énfasis que pone en los recursos y en su uso sustentable, sin embargo sigue el debate, y aunque algunos Estados lo han incluido en sus legislaciones (al igual que el de Seguridad Alimentaria), no ha sido aceptado por todos los países miembros de Naciones Unidas. ${ }^{20}$

19 El Comité de Derechos Económicos, Sociales y Culturales de Naciones Unidas es un órgano independiente y está conformado por 18 miembros, tiene a cargo el monitoreo e interpretación del Pacto, específicamente se expidió sobre el Derecho a una Alimentación Adecuada en la Observación General nº 12.

20 La Ley Marco sobre Derecho a la Alimentación, Seguridad y Soberanía Alimentaria, aprobada en la XVIII Asamblea Ordinaria del Parlamento Latinoamericano de 2012 en Panamá, señala en su Artículo $1^{\circ}$ : "El objeto de la presente Ley es establecer un marco jurídico de referencia, que permita a cada Estado establecer políticas y estrategias para garantizar de manera permanente y con carácter de prioridad nacional "El Derecho a la Alimentación", la seguridad alimentaria y nutricional de la población, para el disfrute de una vida sana y activa. Los Países que hayan adoptado el concepto de Soberanía adecuarán el objeto de la presente Ley". Esta ley-modelo ha sido adoptada en el marco de FAO y como podemos derivar de la cita anterior, no adscribe a la Soberanía Alimentaria como un concepto a seguir. 
Los Estados que conciben a la Soberanía Alimentaria dentro de sus legislaciones son: Argentina en su Acuerdo de Cooperación en materia de Soberanía y Seguridad Alimentaria con la República de Venezuela, este último también posee su propia Ley Orgánica; Bolivia en la Ley de Revolución Productiva Comunitaria Agropecuaria; Ecuador tiene la Ley Orgánica del Régimen de la Soberanía Alimentaria; y por último, Nicaragua en su Ley ${ }^{\circ}$ 693. Se advierte que todos ellos son países en desarrollo y que la asimilación del concepto no ha conllevado un aseguramiento real que asegure tanto la Soberanía como la Seguridad Alimentaria.

De allí que la Seguridad Alimentaria, como veremos, es un concepto más neutro y es el que se ha utilizado como base en la construcción de políticas alimentarias, programas que trabajan para la erradicación del hambre y que siguen los lineamientos FAO.

\section{Aproximaciones a la 'Seguridad Alimentaria'}

En 1974 fue convocada la Conferencia Mundial de la Alimentación en razón de la preocupante situación de desnutrición generalizada y la necesidad de cubrir las futuras demandas alimentarias. En ese marco se adoptó la Declaración Universal sobre la erradicación del hambre y la malnutrición, en la cual se reconoció el Derecho inalienable de las personas a no padecer hambre, con el fin de que éstas puedan desarrollarse plenamente, siendo tarea de todos los Estados garantizarlo.

Allí surgió la idea de la Seguridad Alimentaria, definida como "[...] un sistema mundial de seguridad alimentaria que asegure la disponibilidad suficiente de alimentos a precios razonables en todo momento, independientemente de las fluctuaciones y caprichos periódicos del clima y sin ninguna presión política ni económica" (punto g). ${ }^{21}$ Esta noción fue la primera que apareció, sin embargo ya en el año 2002 existían más de doscientas definiciones en la literatura. ${ }^{22}$

A lo largo de los años, el concepto ha ido adquiriendo nuevos matices que enriquecieron sus alcances ${ }^{23}$. Los principales dan cuenta que en 1983 la

21 La Declaración fue aprobada el 16 de noviembre de 1974 por la Conferencia Mundial de la Alimentación, convocada por la Asamblea General en su resolución 3180 (XXVIII) de 17 de diciembre de 1973; y que hizo suya la Asamblea General en su resolución 3348 (XXIX) de 17 de diciembre de 1974.

22 Toma -Tbianov y Saramet (2012) p. 153.

23 Clay (2002) p. 27. 
FAO agrega la idea de garantizar el acceso físico y económico de alimentos; en 1986 el Banco Mundial opta por otro, que incluye un aspecto nuevo que consiste en proveer alimentos suficientes para llevar una vida activa y saludable. Años después, en 1996, con motivo de la Cumbre Mundial de Alimentación, el concepto se complejiza, sumando que la Seguridad Alimentaria debe darse a nivel individual, familiar, nacional, regional y mundial. Como se advierte la noción se ha ido perfeccionando, incluyendo aspectos económicos y culturales, hasta llegar a la actual, que incluye la inocuidad y nutrición.

La definición más comúnmente aceptada hoy y que vemos se mantiene en textos de la FAO, explica que la Seguridad Alimentaria es: "La situación que se da cuando todas las personas tienen, en todo momento, acceso físico, social y económico a suficientes alimentos inocuos y nutritivos para satisfacer sus necesidades alimenticias y sus preferencias en cuanto a los alimentos a fin de llevar una vida activa y sana". ${ }^{24}$

Esta concepción comprende cuatro dimensiones, a saber: "la disponibilidad de los alimentos, su acceso físico y económico, la utilización que se hace de ellos para cubrir necesidades biológicas y la estabilidad a lo largo del tiempo". ${ }^{25}$ A pesar de la existencia de una conceptualización, en cuanto a su aplicación los autores destacan que se entiende y se utiliza de diferentes formas, según el contexto y la región geográfica estudiada. ${ }^{26}$

La situación contraria es la inseguridad alimentaria y las causas de ella son múltiples: no disponibilidad de alimentos, poder adquisitivo insuficiente, entre otras; puede darse de manera crónica, estacional o transitoria. ${ }^{27}$ En la actualidad, hay muchos Estados que siguen padeciendo inseguridad alimentaria crónica, con altos niveles de desnutrición y hambre producto de crisis económicas y desastres naturales; ésta se caracteriza por ser a largo plazo, persistente, en que las personas no tienen capacidad de satisfacer necesidades básicas alimenticias.

En el caso de nuestra América Latina, no hay homogeneidad respecto a la Seguridad Alimentaria de los distintos Estados. No obstante, los 33 países de

24 Seguimos a Rodríguez-Chaves y Pérez (2015) p. 51. Agregamos que para algunos autores, el concepto FAO de Seguridad Alimentaria indica que se trata de la capacidad de suministro de alimentos a una población en cantidad suficiente y en condiciones adecuadas, también entendida como seguridad de suministro.

25 Cfr. FaO et al. (2015) p. 58.

26 BABU et al. (2014) p. 7.

$27 \quad$ FaO et al. (2015) p. 58. 
la región agrupados en la CELAC (Comunidad de Estados Latinoamericanos y Caribeños) aprobaron en enero de 2015 el Plan para la Seguridad Alimentaria, la nutrición y erradicación del hambre para 2025, el cual fue propuesto por FAO, CEPAL (Comisión Económica para América Latina) y ALADI (Asociación Latinoamericana de Integración). ${ }^{28}$ Este plan comprende las dimensiones del concepto de Seguridad Alimentaria y se estructura en base a 4 pilares:

A) Estrategias coordinadas de Seguridad Alimentaria, a través de políticas nacionales y regionales: entre ellas, fortalecer marcos jurídicos, facilitar el comercio y crear programas de abastecimiento de alimentos.

B) Acceso oportuno y sostenible a alimentos inocuos a todas las personas: dentro de esto, se comprenden programas de transferencias condicionadas $^{29}$, crear trabajos estables, fortalecer agricultura familiar.

C) Bienestar nutricional y aseguramiento de nutrientes: con programas que pongan énfasis en niños, mujeres en edad fértil y adultos mayores.

D) Producción estable de alimentos ante desastres naturales.

La región de América Latina y el Caribe ya ha cumplido uno de los Objetivos de Desarrollo del Milenio ${ }^{30}$, el cual fue reducir el porcentaje de subalimentación a 5,5\%, otros países incluso poseen tasas menores al 5\%. Lo anterior, se debe al trabajo que han venido realizando los Estados ${ }^{31}$ adoptando medidas que se enmarcan dentro los pilares ya mencionados, entre ellos, programas agrícolas y de riego, leyes de promoción de alimentación saludable, etiquetado de los alimentos, etc.

28 CEPAL et al. (2016) p. 9.

29 Estos programas buscan disminuir brechas de pobreza a través de transferencias en dinero a familias vulnerables, condicionadas por acceso a otras formas de desarrollo de capacidades o habilidades.

30 La Campaña del Milenio de las Naciones Unidas se inició en 2002 para lograr el apoyo y la inspiración de modo de avanzar en los Objetivos de Desarrollo del Milenio, ocho objetivos entre los que destacamos el Objetivo 1: Erradicar la pobreza extrema y el hambre; y el Objetivo 7: Garantizar la sostenibilidad del Medio Ambiente. Más información en "Podemos erradicar la pobreza. Objetivos de desarrollo del milenio y más allá de 2015", Departamento de Información Pública de las Naciones Unidas, sine data. Disponible en: <http://www.un.org/es/millenniumgoals/bkgd.shtml>, fecha de consulta: 2 julio 2016.

31 Para ver avances por países de la región ingresar a "Plataforma de Seguridad Alimentaria y Nutricional", FAO, AlADI y CEPAL, 2017. Disponible en: <http://plataformacelac.org/>, fecha de consulta: 2 marzo 2017. 


\section{EN CONTEXTOS DE CAMBIO CLIMÁTICO}

Desde el origen de la tierra, el clima ha experimentado variaciones fundamentalmente por causas naturales (erupciones, cambios en órbitas planetarias y en placas tectónicas) ${ }^{32}$. Junto a ello, el aumento de la población y los procesos de industrialización han incrementado la emisión de Gases de Efecto Invernadero (GEI), generando así el fenómeno de Cambio Climático. Los GEI permiten el desarrollo de la vida en el planeta al retener parte de la energía solar en la tierra (efecto invernadero natural). Entre estos gases se encuentra el dióxido de carbono (CO2), el metano $(\mathrm{CH} 4)$, óxido nitroso $(\mathrm{N} 2 \mathrm{O})$, hexafluoruro de azufre (SF6), hidrofluorocarbono (HFC) y perfluorocarbono (PFC), y otros. Algunos de ellos se liberan a la atmósfera como parte de procesos naturales, mientras que otros son compuestos puramente industriales. ${ }^{334}$

A continuación, aportaremos algunas informaciones del fenómeno climático, para revisar brevemente el modo en que la comunidad internacional ha asumido el problema.

\section{Breves referencias al Cambio Climático}

Los últimos años han sido testigos de que la concentración de GEl ha llegado a niveles nunca antes vistos. Este incremento es superior a la capacidad de remoción natural que tienen, elevando así la temperatura de la superficie terrestre en $0,85^{\circ} \mathrm{C}$ promedio durante el periodo de 1880 a $2012 .{ }^{35}$

Este fenómeno, conocido como calentamiento global, es la manifestación más visible del Cambio Climático, cuyos efectos se expresan en los diferentes subsistemas del Sistema Climático, es decir, en la atmósfera, hidrosfera (océanos), litósfera (continentes), biosfera (flora y fauna), criósfera (hielos y nieve), los que funcionan como un todo que se autorregula con tiempos de respuesta que varían de uno a otro, por lo que un desequilibrio en uno de ellos implicará consecuencias en todo el sistema. ${ }^{36}$

Según el último informe del PICC, las emisiones de GEl aumentaron más rápidamente entre 2000 y 2010 que en cada uno de los tres decenios anteriores. Las emisiones de $\mathrm{CO}_{2}$ por quema de combustibles fósiles y

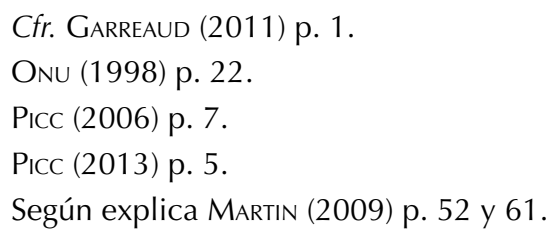


procesos industriales, contribuyeron en alrededor del $78 \%$ al alza de emisiones de GEI totales de 1970 a $2010 .^{37}$

De acuerdo al último informe del PICC ${ }^{38}$, se observan precipitaciones cambiantes, glaciares en retroceso, derretimiento de nieve y hielo, cambios en el nivel del mar, acidificación del mar, todo lo que está alterando los sistemas hidrobiológicos y generando múltiples consecuencias en los distintos ecosistemas. Asimismo, se generan fenómenos climáticos extremos, tales como olas de calor, sequías, inundaciones, ciclones e incendios forestales, entre otros.

Por ello puede afirmarse que los cambios de clima causan impactos en los sistemas humanos ${ }^{39}$ y naturales, pero es en estos últimos donde los efectos son aún más evidentes.

Los efectos del Cambio Climático operan de distintas formas en los sistemas humanos. Destacamos los daños a la infraestructura por fenómenos climáticos extremos; pérdidas y mermas en los sistemas productivos como agricultura (por sequías), acuicultura y pesca (por cambios en el nivel del mar y acidificación); el déficit de agua, que afectará el consumo de este recurso, como también a la generación de electricidad; la salud humana será vulnerable a través de distintos mecanismos ${ }^{40}$, entre ellos: se prevén episodios críticos de contaminación, olas de calor y es probable que se prolonguen las estaciones de transmisión de importantes enfermedades transmitidas por vectores, se altere su distribución geográfica, llegando enfermedades a sitios donde no se presentaban antes e incluso reemergiendo enfermedades zoonóticas ${ }^{41}$, sin embargo estos últimos impactos sobre la salud humana aún no están bien cuantificados según lo dicho por el PICC.

$37 \quad$ Picc (2015) p. 5.

38 Nos referimos al Informe "Cambio Climático 2013: Bases físicas". Pıcc (2013) p. 5, uno de los cuales fue conformando la información total del Quinto Informe de 2014.

39 Al referirse a los impactos del Cambio Climático no solo hace referencia al medio ambiente, sino también incluye aspectos económicos (por quiebre de los modos tradicionales de producción y costos del Cambio Climático) y de bienestar social (desempleo, pobreza, enfermedades, vulnerabilidad climática).

Así, el Cambio Climático "pone en peligro las vidas y medios de subsistencia de todos los pueblos, y en particular de las personas más vulnerables y desfavorecidas de los países en vías de desarrollo". MARENGo (2010) p. 146.

40 Organización mundial De La Salud (2016) p. 7.

41 Oyhantçabal et al. (2010) p. 175-176. 


\section{Rasgos del Sistema de Protección Internacional}

Al amparo de la Convención Marco de Naciones Unidas sobre el Cambio Climático (CMNUCC) ${ }^{42}$, los Estados aunaron sus fuerzas por estabilizar los Gases de Efecto Invernadero a un nivel que evitara obstrucciones peligrosas con el Sistema Climático, de manera compatible con el desarrollo económico sostenible y de modo que se asegure que la producción de alimentos no se vea amenazada.

El Panel Intergubernamental de Cambio Climático (PICC), como órgano que provee la información científica del fenómeno, ha generado hasta la fecha cinco reportes, el primero en el año 1990, el segundo en 1995, el tercer Informe fue emitido en el año 2001, el cuarto Reporte es del año 2007. El último informe de 2014, el quinto informe técnico, inicia con la siguiente frase: "La influencia humana en el sistema climático es clara, y las emisiones antropógenas recientes de gases de efecto invernadero son las más altas de la historia. Los cambios climáticos recientes han tenido impactos generalizados en los sistemas humanos y naturales". ${ }^{43}$

La labor del Panel ha nutrido con sus informaciones al Sistema de Protección de Cambio Climático, dando luz al fenómeno de calentamiento, cuyo origen, es indudable, se desprende de la actividad humana sobre el Sistema Climático ${ }^{44}$, y es por ello que en la Convención Marco sobre Cambio Climático (CMCC) de 1992, se define el Cambio Climático como "un cambio de clima atribuido directa o indirectamente a la actividad humana que altera la composición de la atmósfera mundial y que se suma a la variabilidad natural del clima observada durante periodos de tiempo comparables" ${ }^{45}$

42 La Convención fue firmada en 1992, en ocasión de la Conferencia de Naciones Unidas sobre Medio Ambiente y Desarrollo que se llevó a cabo en Río de Janeiro. Entró en vigencia dos años después, el 21 de marzo de 1994.

$43 \quad$ Picc (2015) p. 2.

44 Algunos autores dan una explicación más detallada y dicen "El CC es un fenómeno de largo plazo atribuido principalmente a los sistemas económicos, tanto en la generación de emisiones de GEl como en el deterioro de los ecosistemas, con los cuales el sistema climático interactúa. [...] Los procesos de desarrollo económico, después de la revolución industrial, han representado un deterioro generalizado tanto del sistema climático como de las funciones eco sistémicas relacionadas". Mora-Motta y León Rodríguez (2017) p. 172.

45 Onu (1992) p. 3. 
La entrada en vigencia del Protocolo ha sido un tanto accidentada, dado que fue adoptado en Japón en 1997 y recién entró en vigor en febrero del 2005, luego de un largo proceso de ratificación ${ }^{46}$, de similares características que el previsto en el Acuerdo de París (pero cuyo éxito ya se está palpando).

Como segundo instrumento del Sistema del Protocolo de Kioto, destacamos dos puntos relevantes: el primero es que los países desarrollados asumen compromisos cuantificados de limitación y reducción de las emisiones del período 2008 al 2012 (los que están extendidos hasta el 2020), por ende no hay obligaciones legales para los países en desarrollo, contando con un tratamiento diferenciado a su favor; el segundo punto tiene que ver con que añade herramientas para abaratar los costos de mitigación, creando tres mecanismos de mercado para intercambiar créditos de carbono, los llamados mecanismos flexibles, a saber: Aplicación Conjunta (AC), Mecanismo de Desarrollo Limpio (MDL, el mecanismo importante para países en desarrollo) y el Comercio de Emisiones de Carbono (donde la experiencia de la Unión Europea es la más relevante).

Inmediatamente vigente el Protocolo de Kioto, los Estados Partes estuvieron concentrados en las negociaciones climáticas para reemplazar el Protocolo, dado que el sistema enfrentaba el gran desafío de alcanzar un acuerdo internacional que avanzara en los compromisos; mientras, se fueron adoptando distintas decisiones que fueron implementando el Protocolo de Kioto. Las negociaciones lograron el cometido en la Conferencia de las Partes de la Convención Marco de Naciones Unidas sobre el Cambio Climático de 2015 (COP 21) en París.

\section{Las primicias del Acuerdo de París}

En este ítem nos ocuparemos de los puntos más relevantes del Acuerdo de París, el acuerdo multilateral ambiental más exitoso en cuanto a su entrada en vigor, la que ha sucedido en tiempo récord, en poco más de cinco meses, alcanzándose el mínimo de adhesiones para asegurar su vigencia desde el 4 de noviembre de 2016, y ya está funcionando con su primer período de sesiones de la Conferencia de las Partes en Marrakech del 7 al 18 de noviembre de $2016 .{ }^{47}$

46 El Protocolo de Kioto requería de 55 Estados Partes (incluidos Estados del Anexo 1) cuyas emisiones de GEl equivalieran al 55\% del total de emisiones de $\mathrm{CO}^{2}$ de las de los países desarrollados al año 1990.

$47 \quad$ El 5 de octubre de 2016 el Secretario General de las Naciones Unidas anunció que ese día se cumplían las condiciones para la entrada en vigor del Acuerdo de París y que en- 
Desde este Acuerdo, se impulsan los esfuerzos para limitar el aumento de la temperatura por debajo de 1,5 grados centígrados, y se acordó la mantención del aumento de la temperatura global por debajo de los $2^{\circ} \mathrm{C}$. Este ya es un objetivo global de cumplimiento exigible por el Derecho Internacional.

Desde el inicio del Acuerdo se ratifica la noción de Desarrollo Sostenible y su íntima relación con el Cambio Climático, donde lo económico, lo social y lo ambiental deben combinarse en cualquier estrategia para enfrentar el fenómeno del calentamiento, ya que sería un error hacerlo solo desde una visión ambiental o desde una perspectiva económica.

En el Acuerdo, se destaca la importancia que se otorga tanto a la mitigación como a la adaptación y las acciones domésticas se revalorizan; está claro que la mitigación no es suficiente para enfrentar el fenómeno y que mayores costos de adaptación pueden ser necesarios si no hay mitigación (ver artículo 7, punto 4).

Un destacable avance del Acuerdo es que obliga de igual modo a todas las Partes a presentar sus Contribuciones Determinadas a Nivel Nacional (CDN), lo que deberán realizar cada cinco años; estas Contribuciones representarán un avance con respecto a las obligaciones asumidas por esa Parte hasta la fecha (un progreso), cada vez que las presentan, es decir deben incluir medidas más intensas y ambiciosas adoptadas en el marco de las obligaciones anteriores de ese Estado o Parte. Estos compromisos permitirán que las Partes le den seguimiento a sus emisiones y registren sus progresos, los que deberán ir enfocados en la disminución de las emisiones de GEl.

Esta equiparación en los compromisos de las Partes, se apoya en que los países desarrollados aumenten la prestación de un apoyo previsible a las Partes, que son países en desarrollo, en forma de financiación, tecnologia y fomento de la capacidad. La contracara de igualar en las obligaciones a los países en desarrollo, reafirma que requieren asistencia para enfrentar acciones de mitigación y adaptación a largo plazo, incluso distingue a los países menos adelantados y a los pequeños Estados insulares en desarrollo.

traría en vigor el 4 de noviembre de 2016 (Cfr. "Preguntas y respuestas sobre temas jurídicos y de procedimiento relativos a la convocatoria del primer período de sesiones de la Conferencia de las Partes en calidad de reunión de las Partes en el Acuerdo de París", Secretaría de la CMNUCC, 13 de octubre de 2016. Disponible en: < http://unfccc.int/files/ meetings/marrakech_nov_2016/application/pdf/questions_and_answers_on_cma_1_esp. pdf $>$, fecha de consulta: 2 julio 2016). Hoy cuenta con más de 110 Estados Partes (de los 197 que son parte en la Convención Climática). 
Como parte del Acuerdo, los Estados tienen la obligación de realizar un balance global periódico del inventario de emisiones, que evalúa los progresos a largo plazo en el marco de los objetivos del Acuerdo; estando previsto que el primer balance global se realizará en 2023 y luego cada cinco años.

Un punto interesante que se incluyó, tiene que ver con el Mecanismo Internacional de Varsovia para Pérdidas y Daños; es positivo que el Acuerdo asuma que el Cambio Climático genera pérdidas y daños asociados a sus efectos adversos, incluidos los fenómenos meteorológicos extremos y los fenómenos de evolución lenta. Y que el propio Sistema Climático obligue a todos los Estados a contar con sistemas de alerta temprana, a prepararse para situaciones de emergencia, e incluso abre la posibilidad de que estos sistemas preventivos funcionen de manera coordinada por varios Estados miembros del Acuerdo (por ejemplo, por regiones). Hay un reconocimiento del riesgo que el Cambio Climático significa para los Estados y también que la resiliencia es parte de lo que deben ocuparse.

Algo que se está instalando en la comunidad internacional, tiene que ver con la necesidad de que en el tratamiento de estos problemas se asegura la participación del sector público y privado para la implementación de las medidas nacionales.

En otro orden, el Acuerdo destaca al financiamiento como uno de sus elementos prioritarios; se ordena además que el mecanismo de financiamiento de la Convención Climática se haga extensivo a este Acuerdo (artículo 9). ${ }^{48}$ Sin financiamiento, se ve lejana tanto la mitigación como la adaptación al Cambio Climático.

Como uno de los grandes temas que requieren de una implementación relevante, la transferencia de tecnología se alza como un gran desafío para el Acuerdo, tanto para mejorar la resistencia al Cambio Climático como para reducir las emisiones de GEI. ${ }^{49}$

Sin menguar en la importancia de la implementación como una característica clásica del Derecho Ambiental, el Acuerdo se concibe en un marco de

48 La Convención Climática aborda esto en el artículo 21, párrafo 3 (Disposición Provisional), prevé que el Fondo para el Medio Ambiente Global (PNUMA) y el Banco Internacional de Reconstrucción y Desarrollo (BIRD) serán las instituciones que provisionalmente deben asegurar el mecanismo previsto en el artículo 11.

49 La transferencia de tecnología toca muchos temas relacionados con el comercio internacional, como propiedad intelectual, subsidios, bienes ambientales, etc. 
construcción de confianzas mutuas de modo transparente, cuyo objetivo último es promover la aplicación efectiva; se instituye un mecanismo para facilitar la aplicación y promover el cumplimiento de las disposiciones del Acuerdo, que tendrá un cariz técnico, pero que considerará las condiciones nacionales, de una manera que sea transparente, no confrontacional ni punitivo (artículo 15).

Subrayamos que el Acuerdo reconoce una multiplicidad de niveles para enfrentar la problemática del calentamiento, desde el nivel local, sub-nacional, nacional, regional e internacional. ${ }^{50}$

Los primeros pasos de implementación del Acuerdo a nivel internacional se han iniciado, la Proclamación de Acción de Marrakech explica que está en marcha "[...] el comienzo de una nueva era de implementación y acción para abordar el Cambio Climático y conseguir un desarrollo sostenible". La Proclamación reconoce a la acción climática en multiplicidades de foros para responder al problema, y se habla de solidaridad, de actores no estatales, de grandes desafíos de desarrollo, etc. ${ }^{51}$

En suma, el Acuerdo presenta una visión más holística de las estrategias para enfrentar a largo plazo el Cambio Climático a nivel global; advertimos que suma los mecanismos y acuerdos negociados en las conferencias anuales, y marca un nuevo punto de partida en la cooperación climática que requerirá decisiones para la implementación. Revisaremos las principales conexiones de Cambio Climático y la Seguridad Alimentaria.

50 Artículo 7 Punto 2. Las Partes reconocen que la adaptación es un desafío mundial que incumbe a todos, con dimensiones locales, sub-nacionales, nacionales, regionales e internacionales, y que es un componente fundamental de la respuesta mundial a largo plazo frente al cambio climático y contribuye a esa respuesta, cuyo fin es proteger a las personas, los medios de vida y los ecosistemas, teniendo en cuenta las necesidades urgentes e inmediatas de las Partes que son países en desarrollo particularmente vulnerables a los efectos adversos del cambio climático.

51 La "Marrakech Action Proclamation for our Climate and Sustainable Development" decidió en la sesión plenaria de la Conferencia de las Naciones Unidas sobre el Cambio Climático (COP22 del 7 al 18 de noviembre de 2016). Consultar en "La Proclamación de Acción de Marrakech establece un proceso irreversible de acciones climáticas", ONU Cambio Climático Noticias, sine data. Disponible en: < http://newsroom.unfccc.int/es/ noticias/la-proclamacion-de-accion-de-marrakech-establece-un-proceso-irreversible-deacciones-climaticas/>, fecha de consulta: 2 de diciembre de 2016. 


\section{INTERRELACIONANDO CAMBIO CLIMÁTICO Y SEGURIDAD ALIMEN- TARIA}

Los impactos que hemos relatado que tendrá el Cambio Climático, afectarán a todas las regiones del planeta en mayor o menor medida, según la vulnerabilidad que posean. La temática está siendo abordada por distintos organismos internacionales, entre ellos destacamos a la FAO, la Organización Mundial de la Salud y la CEPAL, quienes a partir de los informes del PICC proporcionan nuevas soluciones y propuestas relativas a cada sector o región por la cual trabajan.

Como ya mencionamos, los sistemas naturales son altamente vulnerables al Cambio Climático y los efectos podrían comprometer seriamente la agricultura, silvicultura y la pesca; las consecuencias no solo serán para cada sector, sino que se perturbará en general la producción de alimentos, junto a ello el avanzar hacia la erradicación del hambre, la desnutrición y alcanzar la Seguridad Alimentaria serán metas más difíciles de alcanzar. Por esta razón, la FAO hace un Ilamado a los Estados para que enfrenten el Cambio Climático desde la perspectiva de la Seguridad Alimentaria.

Es evidente que el Cambio Climático afectará en mayor medida a países en desarrollo, más aún si estos poseen economías dependientes de sistemas agropecuarios; asimismo, la afectación llegará a países que son grandes exportadores de alimentos, por lo que se pronostican efectos que van mucho más allá de una región en particular. Ni que hablar de los Estados Insulares que usualmente dependen de su agricultura, que no solo se ve afectada por el fenómeno climático, sino que se ve reducida en su espacio de desarrollo (se quedan sin terreno).

La agricultura (gran proveedor de alimentos) como actividad, de por sí está subordinada a la variabilidad climática natural. Sumado a ello, el fenómeno climático hará que ésta quede aún más expuesta. ${ }^{52}$

Por esto, la agricultura enfrenta grandes desafíos para adaptarse al Cambio Climático, los que pasan por gestionar de manera integrada el uso de suelos, mejorar la calidad de éstos, crear cultivos que sean más resistentes a

52 En el caso de América Latina, como contribuciones ya atribuibles al Cambio Climático relacionadas a la producción de alimentos se encuentran la mayor vulnerabilidad de los medios de subsistencia de los agricultores indígenas Aymara, aumento de los rendimientos agrícolas y expansión de las zonas agrícolas en la zona suroriental de América del Sur, además de cambios de flujo en el Amazonas, río de la Plata, y degradación de tierras. PICc (2014) p. 32. 
condiciones climáticas adversas, entre otros. Asimismo, la población mundial crece y se requerirá aumentar la productividad de alimentos, sin embargo la tarea para los Estados es doble considerando que la actividad agropecuaria es gran fuente emisora de GEI. A nivel mundial el 13\% de los GEI provienen de la agricultura, y en el caso de América Latina éste promedia el $21 \%$; estos gases son principalmente el metano y el óxido nitroso, los que además tienen efectos en el calentamiento global muy superiores al del dióxido de carbono, por ello deberá gestionarse una agricultura sustentable, con otras tecnologías y acorde a la labor de mitigar la emisión de GEI. ${ }^{53}$

Por lo tanto, su erradicación no solo pasa por aumentar la producción de alimentos y gestionar suelos para cultivos, pues para erradicar el hambre, además de prever y mitigar los efectos del calentamiento global, se deben tomar en cuenta los otros pilares en torno a la Seguridad Alimentaria.

\section{Planteos en Seguridad Alimentaria en el Acuerdo de París}

Dando continuidad a los grandes objetivos del Sistema Climático, el Acuerdo de París desde el Preámbulo incluye una referencia al "desarroIlo sostenible y la erradicación de la pobreza", y luego expresamente reza: "Teniendo presentes la prioridad fundamental de salvaguardar la seguridad alimentaria y acabar con el hambre, y la particular vulnerabilidad de los sistemas de producción de alimentos a los efectos adversos del Cambio Climático [...]" (el destacado es nuestro), se advierte que la Seguridad Alimentaria debe asegurarse de modo especial frente al Cambio Climático.

Por su parte, el Artículo 2 se refiere a los niveles de temperatura por debajo de los 2 grados Celsius con respecto a los niveles preindustriales, y a proseguir los esfuerzos para limitar ese aumento de la temperatura a $1,5^{\circ} \mathrm{C}$ con respecto a los niveles preindustriales. Luego de ese compromiso claro, los Estados se comprometen a: "Aumentar la capacidad de adaptación a los efectos adversos del Cambio Climático y promover la resiliencia al clima y un desarrollo con bajas emisiones de gases de efecto invernadero, de un modo que no comprometa la producción de alimentos" (el destacado es nuestro). ${ }^{54}$

Vemos que el Acuerdo revitaliza a la Seguridad Alimentaria como un objetivo global, con lo cual frente al Cambio Climático los Estados no están

53 RodríGuez et al. (2015) p. 13.

$54 \quad \mathrm{Y}$ de inmediato conecta con el financiamiento: "c) Elevar las corrientes financieras a un nivel compatible con una trayectoria que conduzca a un desarrollo resciliente al clima y con bajas emisiones de gases de efecto invernadero". 
solos frente a ella, están como comunidad internacional comprometidos a cuidar esta finalidad. Otro alcance es que este compromiso multilateral exige de los Estados su inclusión como un objetivo nacional. Es más, puede ser regional y local también. Hay un reconocimiento en el Acuerdo de París a una gobernanza multinivel del Cambio Climático, como mencionamos más arriba.

Uno de los puntos que se indican como beneficiosos para los países en desarrollo, es la inclusión del Mecanismo Internacional de Varsovia para Pérdidas y Daños, que asegura que los daños y pérdidas sufridos por el fenómeno sean motivo de ayudas especiales dentro del Acuerdo. Asimismo, se posicionan los sistemas de alerta temprana que deberán desarrollarse en el marco del Acuerdo, vistos como una herramienta para prevenir y disminuir los impactos en los sistemas agropecuarios.

\section{BREVE RESEÑA DE SEGURIDAD ALIMENTARIA Y CAMBIO CLIMÁTICO EN CHILE}

De lo que venimos reflexionando está claro que el Cambio Climático puede afectar negativamente la Seguridad Alimentaria de un Estado y por ello es necesario considerar sus vulnerabilidades para planear estrategias adecuadas en vistas de los compromisos adoptados.

Sobre las emisiones, en Chile van en aumento y las que provienen de la agricultura representan el $15 \%$ de las emisiones totales. ${ }^{55} \mathrm{El}$ desglose de este $15 \%$, de acuerdo a los datos proporcionados por el Sistema Nacional de Inventarios de GEI (SNI Chile), son los siguientes ${ }^{56}: 52,4 \%$ proviene de suelos agrícolas, un 34,4\% de fermentación entérica, $12,1 \%$ de manejo del estiércol y un $1 \%$ de cultivo de arroz y quema en el campo de los residuos agrícolas. Estos datos varían respecto a los considerados para América Latina y el Caribe, por lo que sería interesante evaluar medidas costo efectivas aplicadas a la realidad de Chile, que intenten disminuir los gases de la agricultura (entendida ampliamente) para Chile, sin que estas comprometan la Seguridad Alimentaria.

55 CEPAL y OCDE (2016) p. 44.

56 "Inventario nacional de gases de efecto invernadero de Chile, serie temporal 1990-2010", Ministerio del Medio Ambiente de Chile y Sistema Nacional de Inventarios de Gases Efecto Invernadero, diciembre 2014. Disponible en: <http://www.snichile.cl/sites/default/ files/documentos/2014_iin_cl.pdf>, fecha de consulta: 8 de diciembre 2016, p. 92. 
Chile, previo al Acuerdo de París, plantea una reducción del 30\% de las emisiones de GEl al año 2030 (respecto a 2007), e incluso de un $45 \%$ de contar con el necesario financiamiento. Es por ello, entre otras cosas, que se trabaja fuertemente en el Plan de Acción Nacional de Cambio Climático (2017-2022). Si bien en dicho documento no se contempla un ítem acerca de la Seguridad Alimentaria, relacionados hay una serie de aspectos a ser considerados y que se irán indicando.

El anteproyecto del Plan de Acción Nacional ha definido nueve sectores prioritarios en los que deben implementarse planes sectoriales ${ }^{57}$. De éstos, los que podrían vincularse directamente a Seguridad Alimentaria son: el silvoagropecuario, pesca y acuicultura, recursos hídricos y salud. Actualmente, solo dos de ellos ya se encuentran aprobados y en proceso de implementación, que corresponden al área silvoagropecuaria y pesca-acuicultura.

En dicho anteproyecto, los estudios en general indican que uno de los sectores más afectados por el Cambio Climático será el silvoagropecuario, en el que se prevé un desplazamiento de cultivos, producto del aumento de temperaturas y erosión del suelo, menor disponibilidad de agua para riego, afectando principalmente a agricultores y ganaderos de la zona norte y central del país. Sin embargo, no todo es negativo, ya que hacia el sur del país sí se esperan mejoras en la productividad.

En el plan sectorial para pesca y acuicultura (ya en fase de implementación), se describen algunos efectos positivos y otros negativos, según la captura de peces de que se trate, ya que algunos podrán encontrarse en mayor o menor disponibilidad según requieran mayores o menores temperaturas. Es posible que el aumento de temperaturas y salinidad del agua pueda afectar a los centros de cultivos (principalmente salmones, crustáceos y moluscos), por aumento de la prevalencia de enfermedades y plagas; contribuyendo a la proliferación de microalgas tóxicas. En definitiva, de los impactos previstos, éstos no solo podrán afectar la producción de alimentos, sino también la calidad de éstos, afectando la nutrición y salud de la población más vulnerable.

Este plan específico para pesca y acuicultura contempla contribuir a la adaptación de las áreas más vulnerables, con la idea de mejorar los

57 El Plan se encuentra como anteproyecto trabajándose en su etapa final. Cfr. "Plan de Acción Nacional de Cambio Climático 2017-2022: anteproyecto en consulta pública", Ministerio del Medio Ambiente, 18 abril 2016. Disponible en: <http://portal.mma.gob. cl/wp-content/uploads/2016/04/Anteproyecto-PANCC-2017-2022-FINAL-2016-04-18. pdf>, fecha de consulta: 7 de diciembre 2016, p. 29. 
beneficios socio-económicos que entrega el sector y de resguardar la Seguridad Alimentaria y biodiversidad acuática. Es más, se señala expresamente, en el que podrán verse afectadas las cuatro dimensiones de la Seguridad Alimentaria. ${ }^{58}$ Son las únicas medidas que se han tomado y que hacen referencia expresa a la Seguridad Alimentaria como una finalidad de estas políticas sectoriales.

En el caso del sector silvoagropecuario (plan en vigencia), se observa que es el Ministerio de Agricultura, siguiendo la línea de fomentar una agricultura sustentable, quien es el responsable de implementar el plan sectorial a través de regulaciones y programas que comprenden transferencia de tecnología, innovación y otorgamiento de apoyo financiero a pequeños productores. ${ }^{59}$

Cabe destacar que la CONAF (Corporación Nacional Forestal) es la institución que trabaja contra la desertificación y erosión de los suelos. Algunas medidas concretas que se vienen desarrollando a partir del plan del año 2013 son: programas de mejoramiento genético para cultivos agrícolas, programas para establecer huella del agua, desarrollar un sistema nacional de gestión de riesgos frente a eventos climáticos y emergencias agrícolas, instrumentos de seguro agrícola frente a cambios climáticos, incentivos a la sustentabilidad, entre otras. ${ }^{60}$

58 Para ello, se trabaja en la implementación de manejo de pesqueras, determinar áreas aptas para acuicultura en base a los posibles escenarios climáticos, fortaleciendo otros programas ya existentes, buscando mecanismos de financiamiento, creación de incentivos y desincentivos para las buenas prácticas, entre otros. Cfr. "Plan de Adaptación al Cambio Climático para pesca y acuicultura", Subsecretaría de pesca y acuicultura, Ministerio de Economía, Fomento y Turismo y Ministerio del Medio Ambiente de Chile, 2015. Disponible en: <http://portal.mma.gob.cl/wpcontent/uploads/2016/12/Plan-Pesca-y-AcuiculturaCMS.pdf>, fecha de consulta: 19 de diciembre 2016, p. 18.

59 Cfr. "Plan de Acción Nacional de Cambio Climático 2017-2022: anteproyecto en consulta pública", Ministerio del Medio Ambiente, 18 abril 2016. Disponible en: <http:// portal.mma.gob.cl/wpcontent/uploads/2016/04/Anteproyecto-PANCC-2017-2022-FINAL-2016-04-18.pdf>, fecha de consulta: 7 de diciembre 2016, pp. 15 y 33.

60 Estas son algunas de las medidas que se enmarcan en las líneas de acción; son cinco, a saber: mejorar la competitividad de la agricultura, fomento a la investigación e innovación, promover la sustentabilidad económica, social y ambiental, acceso al mercado y por último modernizar el Ministerio de Agricultura y sus servicios. Cfr. "Plan de Adaptación al Cambio Climático del sector silvoagropecuario", Ministerio de Agricultura y Ministerio del Medio Ambiente de Chile, octubre de 2013. Disponible en: <http://www.mma.gob. cl/1304/articles-55879_InstrumentoFinalCC_Silvoagropecuario.pdf $>$, fecha de consulta: 8 de diciembre 2016. 
En salud, aún se trabaja en elaborar un plan, no obstante en el documento Plan de Adaptación Nacional del período 2008-2012 ${ }^{61}$ publicado el año 2015 se dan ciertas luces de algunos de los ejes que se deben seguir, ello porque el Cambio Climático alterará pilares de la salud humana, dadas las variaciones en la calidad de los alimentos y la disponibilidad de agua, por lo que una línea de acción debe orientarse a implementar medidas frente a los eventos extremos que puedan contaminar nuestros alimentos con el objeto de que la población pueda contar con un abastecimiento estable.

En general, puede decirse que no queda claro aún el papel de la Oficina de Cambio Climático en estas medidas, ni tampoco la participación de los ministerios relacionados, tampoco cuáles serán las medidas de monitoreo y seguimiento.

Exploradas estas primeras medidas que se están tomando en la materia, podemos advertir que en Chile se está avanzando en fijar las estrategias para enfrentar el Cambio Climático, hay conciencia que la agricultura requiere la atención renovada de las políticas públicas y esperamos que haya una mayor mirada hacia la Seguridad Alimentaria que asegure los cuatro pilares y que se coordine con las otras medidas que Chile debe tomar para asegurar la mitigación en las emisiones de GEI y la necesaria adaptación de los sistemas naturales y humanos.

\section{PROPUESTAS DE MEDIDAS EN EL MARCO DEL CAMBIO CLIMÁTICO}

Para cerrar este trabajo, nos gustaría sugerir algunas medidas que permitirían a los Estados ir cumplimentando sus compromisos frente al Acuerdo de París y la Seguridad Alimentaria, siguiendo las cuatro dimensiones, a saber:

\section{Sobre la disponibilidad de los alimentos}

A) Desarrollar la agricultura sostenible y aumentar productividad, e incentivar a productores (debería pensarse en subsidios, por ejemplo);

B) Desarrollar métodos de cooperación entre Estados (aprovechar ventajas y desventajas de los efectos del fenómeno climático entre las regiones, y también a nivel nacional) ${ }^{62}$;

61 "Plan de Adaptación al Cambio Climático", Ministerio del Medio Ambiente de Chile, 2015. Disponible en: <http://portal.mma.gob.cl/wp-content/uploads/2016/02/Plan-Nacional-Adaptacion-Cambio-Climatico-version-final.pdf>, fecha de consulta: 8 de diciembre 2016, p. 61 .

62 Se habla de que el Cambio Climático incrementará el potencial del movimiento de pestes y enfermedades, al tiempo que de productos, de un Estado a otro. De allí el llamado que 
C) Incluir tecnologías y energías limpias;

D) Inversiones de los Estados en I+D (investigación y desarrollo).

\section{Sobre cómo garantizar el acceso a los alimentos}

A) Los Estados deben preparar sus políticas comerciales en relación con los alimentos (exportaciones/importaciones);

B) Reconocer la necesidad del manejo multinivel del acceso a los alimentos (entre Estados, a nivel nacional, regional y local).

\section{En cuanto al consumo de alimentos y su nutrición}

A) Avanzar en los proyectos sectoriales, con especial énfasis en salud;

B) Programas de inocuidad alimentaria transversales por sectores (mejorar trazabilidad de los alimentos en el contexto del comercio internacional, mayor incorporación de estándares internacionales, etiquetado, etc.);

C) Planes de cooperación internacional y regional frente a enfermedades emergentes ligadas a la producción y comercio de alimentos.

\section{Estabilidad de la Seguridad Alimentaria}

A) Trabajar en planes, institucionalidad y coordinación de los distintos organismos nacionales que intervienen en desastres;

B) Gestionar los distintos tipos de riesgos a los que están expuestos, junto con medidas de abastecimiento de alimentos, control sanitario de los alimentos y agua, revisión de planes y medidas inmediatas de mitigación frente a desastres que afecten la agricultura, la industria pecuaria y pequeños productores;

C) Comprender el riesgo en general, y promover las prácticas preventivas que mitiguen los peligros naturales y los desastres;

hace $\mathrm{FAO}$ a fortalecer la cooperación internacional y regional para facilitar el intercambio de conocimientos, el manejo común de recursos y el intercambio de recursos genéticos de plantas y animales. Cfr. FAO (2016) p. 102. Este Reporte de FAO es posterior al Acuerdo de París. 
D) Estudiar nuevos métodos para la producción agropecuaria o reorientar y diversificar la producción de cada Estado, según las ventajas que cada uno posea.

\section{CONCLUSIONES: UNA ÚLTIMA MIRADA DESDE LA GOBERNANZA GLOBAL}

En este trabajo, nos propusimos adentrarnos en la Seguridad Alimentaria como uno de los grandes desafíos de la humanidad que en contextos de Cambio Climático y ante la exitosa partida del Acuerdo de París, el primer gran acuerdo ambiental y multilateral del Siglo XXI, se ven revitalizadas.

En el recorrido que realizamos mostramos los impactos que el Cambio Climático tendrá sobre los sistemas naturales y humanos y su directa relación con la Seguridad Alimentaria. La complejidad del Cambio Climático exige enfatizar las miradas desde la sustentabilidad, entendida como la armonización de los factores políticos, ambientales y económicos.

Una importante observación es que se propicia y apoya una mayor interacción entre la Seguridad Alimentaria y el Cambio Climático, para las acciones que a nivel institucional se están siguiendo, apoyo que se evidencia desde los propios textos de Cambio Climático, y que se enfatiza ante la posibilidad de nuevas medidas de implementación del Acuerdo de París, para lograr avances en Seguridad Alimentaria también.

Creemos que el Acuerdo viene a apuntalar las políticas nacionales para complementar con el fin último de la Seguridad Alimentaria. Asimismo, se plantea como un objetivo global complementario, con lo cual frente al Cambio Climático los Estados no están solos.

El desafío que la Seguridad Alimentaria conlleva para las políticas ambientales, alimentarias, de salud, económicas, energéticas, etc., es enorme, y la coordinación que el Estado deberá emprender y profundizar es muy grande. Ocuparnos brevemente del caso chileno nos permitió ver como aún falta un gran trabajo para abordar el Cambio Climático, de modo de asegurar la Seguridad Alimentaria. Vislumbramos que cada vez es más clara la necesidad de fortalecer la institucionalidad pública que enfrenta al Cambio Climático.

En contextos de Gobernanza Global, se abre un camino para satisfacer la legitimidad contemporánea, donde ya no está solo el Estado, sino que está acompañado por la comunidad internacional, representada por los tradicionales más los nuevos sujetos de la escena internacional; todo lo que va marcando la renovación de la cooperación internacional, la gran herramienta para enfrentar el Cambio Climático del Siglo XXI. 
Pensamos que la lucha contra el Cambio Climático se ha desvanecido de la regulación doméstica, para pasar al campo de la cooperación internacional, y desde allí brindar los lineamientos que luego el derecho nacional deberá asegurar, con considerable libertad para decidir qué hacer para cumplir con sus compromisos. Láncos ${ }^{63}$ indica que este nuevo sistema de ejercicio de autoridad pública ${ }^{64}$, no solo establece relaciones entre instituciones internacionales, organizaciones de integración económica (como la Unión Europea) y gobiernos nacionales, sino que construye una cooperación horizontal entre gobiernos nacionales que se evidencia mucho más al momento de aplicar, por ejemplo, los mecanismos del Protocolo de Kioto que comentamos más arriba (piénsese en el MDL), que además envuelven al sector privado y a la sociedad civil, a observadores, a científicos, etc., que participan del régimen climático de diversa manera.

Coincidimos en que la legitimidad es algo crucial para todo los participantes del Sistema de Protección Climática ${ }^{65}$, y que ésta debe cuidarse en especial al momento de las implementaciones del Acuerdo de París, de modo de que la transparencia, la participación y la revisión estén presentes en los nuevos mecanismos que deberán crearse para hacer realidad los grandes objetivos del Acuerdo y, en definitiva, de la propia Convención. Nuevamente destaca la Seguridad Alimentaria como un gran objetivo a alcanzar.

Sabemos que los desafíos son grandes, pero también que las recompensas de una población protegida valen los esfuerzos.

63 LÁNCOS (2010) pp. 279 y 280.

64 Destacamos el trabajo de Bogdandy, Dann Y Goldmann, que entrecruzan el concepto de Gobernanza Global con el de ejercicio de autoridad pública internacional de modo de dar luz sobre el primero; los autores definen autoridad como la capacidad legal de determinar a otros y reducir su libertad, y este ejercicio es la realización de esa capacidad en particular, que puede ser mediante la producción de estándares o regulaciones o mediante la difusión de informaciones (como rankings). La determinación puede ser obligatoria o no, es obligatoria siempre que modifica una situación legal. Este concepto amplio de autoridad permite incluir distintas situaciones, como las que generan los Estándares de Doble Tributación de la OECD, o el Código de Conducta de Pesca Responsable de la FAO o los reportes PISA. Bogdandy, Dann y Goldmann (2008) p. 1385 y ss.

65 Seguimos a LÁncos en sus conclusiones sobre el Protocolo de Kioto como ejercicio de autoridad pública, pero que hacemos extensibles al Acuerdo de París. Láncos (2010) p. 299. 


\section{BIBLIOGRAFÍA CITADA}

Babu, Suresh, Gajanan, Shailendra y Sanyal, Prabuddha (2014): Food security, poverty and nutrition policy analysis ( $2^{\text {a }}$ edición, Estados Unidos, Elsevier).

CLAY, Edward (2002): "Food security: concepts and measurement", en Organización De Las Naciones Unidas Para La Alimentación Y La Agricultura (FAO), Trade reforms and Food Security: Conceptualising the Linkages (Roma, FAO) pp. 25-34.

Comisión Económica Para América Latina y El Caribe (CEPAL) y Organización Para La CoOperación y El Desarrollo EConómicos (OCDE) (2016): Evaluaciones del desempeño ambiental: Chile 2016 (Santiago, CEPAL/OCDE).

Comisión EConómica para América Latina y El Caribe (CEPAL), Organización de las

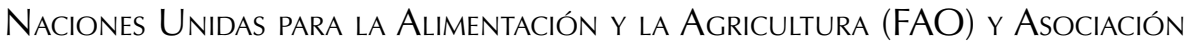
LATINOAMERICANA de INTEGRACIÓN (ALADI) (2016): "Seguridad alimentaria, nutrición y erradicación del hambre. CELAC 2025: Elementos para el debate y la cooperación regionales". Disponible en: <http://repositorio. cepal.org/bitstream/handle/11362/40348/1/S1600707_es.pdf>, fecha de consulta: 12 de marzo 2017.

Cottier, Thomas, Aerni, Philipp, Karapinar, Baris, Matteottı, Sofya, De Sèpibus, Joëlle, y SHINGAL, Anirudh (2014): "The Principle of Common Concern and Climate Change", Archiv des Völkerrechts, Bd. 52: pp. 293-324.

FAO (2016): The state of food and agriculture. Climate Change, agriculture and foodsecurity (Rome, FAO).

FaO, FidA (Fondo Internacional de Desarrollo Agrícola) y PMA (Programa Mundial de Alimentos) (2015): "El estado de la inseguridad alimentaria en el mundo 2015. Cumplimiento de los objetivos internacionales para 2015 en relación con el hambre: balance de los desiguales progresos". Disponible en: <http://www.fao.org/3/a-i4646s.pdf>, fecha de consulta: 5 de diciembre 2016.

Garreaud, René (2011): "Cambio Climático: Bases Físicas e Impactos en Chile", Revista Tierra Adentro, n 93: pp. 13-19.

Gordillo, Gustavo y MÉnDEZ, Obed (2013): "Seguridad y Soberanía Alimentaria (Documento base para discusión)". Disponible en: <http://www.fao. org/3/a-ax736s.pdf>, fecha de consulta: 8 de marzo 2017. 
LÁNCOS, Petra (2010): "Flexibility and legitimacy - The emissions trading system under the Kyoto Protocol" in Von Bogdandy, Armin; Wolfrum, Rüdiger; Von Bernstorff, Jochen; Dann, Philipp y Goldmann, Matthias (eds.) The exercise of Public Authorityby International Institutions (Beiträge zum ausländischen öffentlichen Recht und Völkerrecht 210, Heidelberg, Springer) pp. 271-299.

Mangas, Araceli (2014): Humanización, Democracia y Estado de Derecho en el ordenamiento internacional (Madrid, Real Academia de Ciencias Morales y Políticas).

Marengo, Melissa (2010): "Cambio Climático y la Gran Transformación del Siglo XXI: Hacia un Futuro Sostenible", Derecho y Sociedad, n³5: pp. 146-155.

MARTIN, Javier (2009): "Conceptos previos y conceptos nuevos en el estudio del Cambio Climático reciente", Investigaciones geográficas, n²9: pp. 51-63.

Mora-Motta, Alejandro y LeÓn Rodríguez, Nohra (2017): "Cambiando de perspectiva en la economía de la mitigación del Cambio Climático", Cuadernos de Economía, vol. 36, n70, pp. 169-195.

Ocampo, José (Editor) (2015): Gobernanza global y desarrollo: Nuevos desafíos y prioridades de la cooperación internacional (Buenos Aires, Siglo Veintiuno Editores y Cepal).

Organización De las Naciones Unidas Para la Alimentación y La Agricultura (FAO, 2012). Disponible en: <http://www.fao.org/3/a-au351s.pdf>, fecha de consulta: 4 de abril 2017.

Organización Mundial de la Salud (2016): "Género, Cambio Climático y Salud". Disponible en: <http://apps.who.int/iris/bitstre am/10665/204178/1/9789243508184_spa.pdf>, fecha de consulta: 6 de diciembre 2016.

Ost, François (2006): "La auto-organización ecológica de las empresas: ¿Un juego sin conflictos y sin reglas?", en Gordillo, José (coordinador) La protección de los bienes comunes de la humanidad (Madrid, Editorial Trotta) pp. 79-106.

Oyhantçabal, Vitale y Lagarmilla, Patricia (2010): “El Cambio Climático y su relación con las enfermedades animales y la producción animal", en 
Conferencia OIE. Disponible en: <http://www.oie.int/doc/ged/D11835. PDF $>$, fecha de consulta: 8 de diciembre 2016.

PiCC (Panel intergubernamental de Expertos sobre el Cambio Climático) (2006): "Directrices del IPCC para los inventarios nacionales de gases efecto invernadero". Disponible en: <http://www.ipcc-nggip.iges.or.jp/ public/2006gl/spanish/pdf/0_Overview/V0_0_Cover.pdf>, fecha de consulta: 5 de diciembre 2016.

(2013): "Cambio Climático 2013: Bases físicas". Disponible en: <http://www.ipcc.ch/pdf/assessment-report/ar5/wg1/WG1AR5_ SummaryVolume_FINAL_SPANISH.pdf $>$, fecha de consulta: 5 de diciembre 2016.

(2014): "Cambio Climático 2014: Impactos, adaptación y vulnerabilidad.Resumen para responsables de políticas". Disponible en: <https:// www.ipcc.ch/pdf/assessment-report/ar5/wg2/ar5_wgll_spm_es.pdf >, fecha de consulta: 5 de diciembre 2016.

(2015): "Cambio Climático 2014: Informe de síntesis". Disponible en: $<$ https://www.ipcc.ch/pdf/assessment-report/ar5/syr/SYR_AR5_FINAL_ full_es.pdf $>$, fecha de consulta: 8 de diciembre de 2016 .

Peters, Anne (2016): "The Refinement of International Law: From Fragmentation to Regime Interaction and Politicization", en Max Planck Institute For Comparative Public Law \& International Law (MPIL), Research Paper No. 2016-19. Disponible en SSRN: <https://ssrn.com/ abstract=2823512orhttp://dx.doi.org/10.2139/ssrn.2823512>, fecha de consulta: 1 de diciembre 2016.

Rodríguez, Adrián, López, Tania, MezA, Laura y Loboguerrero, Ana (2015): Innovaciones institucionales y en políticas sobre agricultura y Cambio Climático: Evidencia en América Latina y el Caribe (SANtiago De ChILE, Comisión ECONÓmica para América latina CEPAL).

Rodríguez-Chaves, Blanca y Pérez, Máximo (2015): "La seguridad alimentaria en el ordenamiento jurídico europeo e interno español. Evolución, regulación y perspectivas ante la PAC 2014-2020", en Menéndez, Angel (dir.), Estudios jurídicos sobre seguridad alimentaria (Madrid, Marcial Pons) pp. 47-85.

Serna De La Garza, José (2009): "Reflexiones sobre el concepto de "gobernanza global" y su impacto en el ámbito jurídico", Biblioteca jurídica 
virtual del Instituto de Investigaciones Jurídicas de la UNAM, pp. 2341. Disponible en: <https://archivos.juridicas.unam.mx/www/bjv/libros/6/2897/3.pdf>, fecha de consulta: 2 de diciembre 2016.

Toma-Bianov, A y Saramet, O. (2012): "The concepts of food security and the right to safe food from the international and european perspective", Bulletin of the Transilvania University of Brasov, serie VII, vol. 5: (54) $n^{\circ} 1$ : pp. 153-159.

Von Bogdandy, Armin, DanN, Philipp y Golddann, Matthias (2008): "Developing the Publicness of Public International Law: Towards a Legal Framework for Global Governance Activities", German Law Journal, vol. $9 \mathrm{n}^{\circ}$ 11: pp. 1375-1400.

\section{NORMAS JURÍDICAS CITADAS}

Convención Marco de Naciones Unidas sobre Cambio Climático, Organización de las Naciones Unidas, 1992. Disponible en: <https:// unfccc.int/resource/docs/convkp/convsp.pdf $>$, fecha de consulta: 8 de diciembre 2016.

Declaración Universal de Derechos Humanos, Organización de las Naciones Unidas, 1948. Disponible en: <http://www.ohchr.org/EN/UDHR/ Documents/UDHR_Translations/spn.pdf>, fecha de consulta: 4 de abril 2017.

Declaración Universal sobre la Erradicación del Hambre y la Malnutrición, Organización de las Naciones Unidas, 1974. Disponible en: <http://www.ohchr.org/SP/ProfessionalInterest/Pages/ EradicationOfHungerAndMalnutrition.aspx $>$, fecha de consulta: 17 de noviembre 2016.

Ley Marco: Derecho a la Alimentación, Seguridad y Soberanía Alimentaria.

Pacto Internacional de Derechos Económicos, Sociales y Culturales, Organización de las Naciones Unidas, 1966. Disponible en: <http:// www.ohchr.org/SP/Professionallnterest/Pages/CESCR.aspx $>$, fecha de consulta: 4 de abril 2017.

Protocolo de Kioto de la Convención Marco de las Naciones Unidas sobre el Cambio Climático, Organización de las Naciones Unidas, 1998. Disponible en: <https://unfccc.int/resource/docs/convkp/kpspan.pdf>, fecha de consulta: 8 de diciembre 2016. 